\title{
Whole-blood antibody tests were not highly sensitive for detecting Helicobacter pylori infection
}

Chey WD, Murthy U, Shaw S, et al. A comparison of three fingerstick, whole blood antibody tests for Helicobacter pylori

infection: a United States, multicenter trial. Am J Gastroenterol 1999 Jun;94:1512-6.

\section{QUESTION: How accurate are whole-blood antibody tests for diagnosing Helicobacter pylori infection?}

Design

Blinded comparison of 3 whole-blood antibody tests with tests based on endoscopic biopsy.

\section{Setting}

3 medical centres in the United States (Ann Arbor, Michigan; Syracuse, New York; and Los Angeles, California).

\section{Patients}

131 patients who were 19-87 years of age (mean age 54 y, 59\% men) and were referred for upper endoscopy. Exclusion criteria were treatment for $H$. pylori infection in the previous year or use of antibiotics or bismuthcontaining compounds in the previous month or a proton-pump inhibitor in the previous 7 days.

Test characteristics for detecting Helicobacter pylori infection*

\begin{tabular}{|c|c|c|c|c|c|}
\hline Diagnostic standards & Tests & $\begin{array}{l}\text { Sensitivity } \\
\text { (95\% Cl) }\end{array}$ & Specificity (CI) & $+\mathrm{LR}$ & $-\mathrm{LR}$ \\
\hline \multirow[t]{4}{*}{ Histologic testing } & FlexPack & $76 \%(62$ to 87$)$ & $79 \%(69$ to 87$)$ & 3.6 & 0.3 \\
\hline & QuickVue & $78 \%$ (64 to 88$)$ & $90 \%(81$ to 96$)$ & 7.9 & 0.2 \\
\hline & AccuMeter & $84 \%$ (71 to 93$)$ & $90 \%(81$ to 96$)$ & 8.5 & 0.2 \\
\hline & RUT & $88 \%(76$ to 95$)$ & $93 \%(85$ to 97$)$ & 11.9 & 0.1 \\
\hline \multirow[t]{3}{*}{ Histologic testing and RUT } & FlexPack & $77 \%(62$ to 89$)$ & $80 \%(69$ to 88$)$ & 3.9 & 0.3 \\
\hline & QuickVue & $82 \%(67$ to 92$)$ & $91 \%(82$ to 96$)$ & 8.8 & 0.2 \\
\hline & AccuMeter & $89 \%(75$ to 96$)$ & $92 \%$ (83 to 97$)$ & 11.1 & 0.1 \\
\hline \multirow[t]{3}{*}{ Histologic testing or RUT } & FlexPack & $73 \%(60$ to 84$)$ & $81 \%(71$ to 89$)$ & 3.9 & 0.3 \\
\hline & QuickVue & $71 \%(58$ to 83$)$ & $91 \%(82$ to 96$)$ & 7.7 & 0.3 \\
\hline & AccuMeter & $79 \%(66$ to 88$)$ & $92 \%$ (83 to 97$)$ & 9.8 & 0.2 \\
\hline
\end{tabular}

${ }^{*} \mathrm{RUT}=$ rapid urease testing. LRs defined in Glossary and calculated from data in article.

\section{Description of tests and diagnostic standards}

The 3 whole-blood antibody tests were FlexPack HP (Abbott Diagnostics, Abbott Park, IL, USA), QuickVue (Quidel Corporation, San Diego, CA, USA), and AccuMeter (formerly HpChek; ChemTrak, Sunnyvale, CA, USA). Antibody testing was done by using whole blood obtained with 2 or 3 fingersticks. The 3 diagnostic standards were histological evidence of $H$. pylori infection in biopsies taken from the body and the antrum of the stomach, positive results with both histological and rapid urease testing (RUT) (excluding 12 patients with discordant histological and RUT results), and a positive result on either histological testing or RUT.

\section{Main outcome measures}

Sensitivity and specificity for detecting $H$. pylori infection.

\section{Main results}

The table shows sensitivities, specificities, and likelihood ratios for tests.

\section{Conclusion}

Whole-blood antibody tests were not highly sensitive for detecting Helicobacter pylori infection.
Source of funding: not stated.

For correspondence: $\mathrm{Dr}$ W D Chey, Universit of Michigan Medical Center,3912 Taubman Center, Box 0362, Ann Arbor, MI 48109, USA FAX 734-936-7392.

\section{COMMENTARY}

Consensus statements in North America and Europe have supported a strategy of "test and eradicate H.pylori" for the management of dyspepsia in the office setting. This strategy benefits patients by breaking the cycle of recurrence of duodenal ulcer disease and decreasing the risk for developing future gastric cancer. ${ }^{1}$ The cost benefit of this strategy lies in avoiding endoscopy; therefore, accurate and reliable non-endoscopic tests for $\mathrm{H}$. pylori are needed. ${ }^{2}$

These studies by Chey and colleagues examine the performance of 2 such tests: whole-blood tests done at the point of care to identify antibodies to $H$.pylori and a ${ }^{13} \mathrm{C}$-urea blood test. The latter is a new technique based on the ${ }^{13} \mathrm{C}$-urea breath test in which ${ }^{13} \mathrm{CO}_{2}$ is released from ingested ${ }^{13} \mathrm{C}$-urea if $\mathrm{H}$. pylori, with its urease enzyme, is present in the stomach. Rather than requiring pre- and postbreath samples, a single blood test can be done 30 minutes after ingestion to identify ${ }^{13} \mathrm{C}$-bicarbonate by mass spectrometry.

Important differences exist between the antibody and urease-based technologies. The whole-blood tests give an immediate result and can be done in 5 to 10 minutes. The ${ }^{13} \mathrm{C}$-urea blood test requires more staff input, a 30 -minute delay before sample collection, and fasting for patients. The sample has to be sent to a central laboratory for analysis, and the result and subsequent therapeutic decision are delayed.

The essential question underlying these 2 studies is whether the additional accuracy of the urea blood test is worth the additional cost. This question has 2 parts. First, what is the difference in performance of the 2 tests in the office setting? Second, what patient-related benefits are obtained by that difference? As Chey and colleagues state, no gold standard exists for identifying $H$. pylori, and most evaluations use a proxy of several reference tests combined. Chey and colleagues' approach is a base-case evaluation that uses histological testing alone with a biopsy-based urease test as an additional reference standard for calculating test performance under the worst and best conditions.

(commentary continued on page 31) 